\title{
Quasiperiodicity and Chaos in Cardiac Fibrillation
}

\author{
Alan Garfinkel,, ${ }^{\star \|}$ Peng-Sheng Chen, ${ }^{\ddagger}$ Donald O. Walter, ${ }^{*}$ Hrayr S. Karagueuzian, ${ }^{\ddagger}$ Boris Kogan, \\ Mikhail Karpoukhin, ${ }^{\sharp}$ Chun Hwang, ${ }^{\ddagger}$ Takumi Uchida, ${ }^{\ddagger}$ Masamichi Gotoh, ${ }^{\ddagger}$ Obi Nwasokwa, ${ }^{\star \star}$ Philip Sager, $\$$ \\ and James N. Weiss* \\ *Department of Medicine (Cardiology), University of California, Los Angeles School of Medicine, Los Angeles, California 90095 ; \\ ${ }^{\ddagger}$ Division of Cardiology, Cedars-Sinai Medical Center, Los Angeles, California 90048; ${ }^{\S}$ West Los Angeles Veterans Administration \\ Medical Center, Los Angeles, California 90024; "Department of Physiological Science, and "Tepartment of Computer Science, \\ University of California, Los Angeles, Los Angeles, California 90095; **Harris Chasanoff Heart Institute, Long Island Jewish Medical \\ Center, New Hyde Park, New York 11042
}

\begin{abstract}
In cardiac fibrillation, disorganized waves of electrical activity meander through the heart, and coherent contractile function is lost. We studied fibrillation in three stationary forms: in human chronic atrial fibrillation, in a stabilized form of canine ventricular fibrillation, and in fibrillationlike activity in thin sheets of canine and human ventricular tissue in vitro. We also created a computer model of fibrillation. In all four studies, evidence indicated that fibrillation arose through a quasiperiodic stage of period and amplitude modulation, thus exemplifying the "quasiperiodic transition to chaos" first suggested by Ruelle and Takens. This suggests that fibrillation is a form of spatio-temporal chaos, a finding that implies new therapeutic approaches. (J. Clin. Invest. 1997. 99:305-314.) Key words: arrhythmia • atrial fibrillation • ventricular fibrillation • death, sudden, cardiac • mathematics
\end{abstract}

\section{Introduction}

Ventricular fibrillation (VF $)^{1}$ is the major cause of sudden cardiac death; atrial fibrillation (AF), while not usually lethal, also causes significant morbidity and mortality. In both types of fibrillation, cardiac electrical activity becomes highly disordered, and the heart ceases to contract coherently. Is this disorder random or deterministic? Although Sir Thomas Lewis stated (1) that during fibrillation, "the pauses betwixt the beats bear no relationship to one another," recent studies of fibrillation have revealed organized behavior, consisting of propagating wavefronts meandering through the myocardium in complex patterns $(2,3)$. High-resolution mapping and other studies have detected local spatio-temporal correlations (3-5) and other evidence of determinism (6) during fibrillation, suggesting that the underlying process is not completely random. It is

Address correspondence to Alan Garfinkel, Department of Medicine (Cardiology), University of California, Los Angeles, 47-123 CHS, Los Angeles, CA 90095-1679. Phone: 310-794-7214; FAX: 310-206-9133; E-mail: alang@lifesci.ucla.edu

Received for publication 17 July 1996 and accepted in revised form 5 November 1996.

1. Abbreviations used in this paper: AF, atrial fibrillation; APD, action potential duration; $(\mathrm{dV} / \mathrm{dt})_{\max }$, maximal rate of rise of the action potential upstroke; VF, ventricular fibrillation.

The Journal of Clinical Investigation

Volume 99, Number 2, January 1997, 305-314 therefore tempting to speculate that fibrillation is deterministic chaos, especially since definitive evidence of chaos has been found in some simpler cardiac arrhythmias (7-9).

This paper presents data suggesting that fibrillation is a form of spatiotemporal chaos arising via a quasiperiodic transition. In this scenario, when an oscillatory process is modulated by several additional oscillatory processes, the system destabilizes and becomes chaotic.

\section{Methods}

The techniques of chaos theory require a physiologically stable preparation unlike ventricular fibrillation in vivo, in which progressive ischemia causes the tissue to deteriorate rapidly. So we studied fibrillation in three stable biological models: chronic human atrial fibrillation, stabilized canine ventricular fibrillation, and fibrillationlike activity in thin sheets of canine and human ventricular epicardium in vitro. In addition, we created a fibrillation-like state in a computer model of cardiac propagation.

Human studies. Five patients underwent clinical cardiac electrophysiology study for clinical indications at Universiy of California, Los Angeles (UCLA) Medical Center, and gave their informed consent for the protocol approved by the UCLA Human Subjects Protection Committee (protocol 91-01-033). Intracardiac recordings were made from the high right atrium using either standard octopolar pacing catheters (1-mm interelectrode distance), or monophasic action potential catheters. Recordings were digitized at $1 \mathrm{KHz}$ and recorded directly to optical disk.

Canine ventricular fibrillation model. The intact, excised heart of a dog was perfused by cross-circulation from a second dog to maintain hemodynamic stability during fibrillation, as described previously (10). Ventricular fibrillation was induced by a short burst of $60-\mathrm{Hz}$ alternating current. Unipolar electrograms recorded from a multielectrode plaque sutured to the epicardial surface of the ventricle were digitized at $1 \mathrm{KHz}$ and recorded to optical disk.

Right ventricular sheet preparation. We adapted the right ventricular epicardial sheet preparation described by Davidenko et al. (11), consisting of a 1-2 $\mathrm{mm}$ thick slice of right ventricular epicardium, $\sim 25$ $\mathrm{mm}$ in length and width, pinned down in a chamber and superfused with oxygenated Tyrode's solution at $37^{\circ} \mathrm{C}$. Using an S1-S2 stimulation protocol similar to that used to induce ventricular fibrillation in the canine ventricle $(12,13)$, a reentrant arrhythmia consistent with a persistent spiral wave was readily induced, but was very susceptible to spontaneous termination and could not be induced to break up into multiple reentrant wavefronts to simulate a fibrillation-like state. However, after treatment with the ATP-sensitive $\mathrm{K}^{+}$- channel agonist cromakalim $(5-10 \mu \mathrm{M})$ to shorten the action potential duration by $30-50 \%$ and reduce wavelength commensurately, a fibrillationlike state could be induced. (Wavelength, defined as the product of action potential duration and conduction velocity, has been shown to be an important determinant of susceptibility to fibrillation [14]). In the presence of cromakalim, transitions from organized periodic reentry to disorganized, irregular reentry were observed, either sponta- 
neously (two preparations) or after pacing (one preparation). An extracellular bipolar electrogram (0.5-mm interpole distance) was continuously recorded on tape and later digitized at $1 \mathrm{KHz}$ and analyzed off line. Activation mapping was performed with a 509 bipolar electrode array (interpolar distance $0.5 \mathrm{~mm}$, interelectrode distance $1.6 \mathrm{~mm}$ ), using a computerized mapping system (E-Map, Auckland, $\mathrm{NZ})$, as described previously $(12,13)$.
Analysis of electrograms. Electrograms were analyzed off-line. Activation spikes were identified in each trace by a computerized peak-detecting algorithm whose parameters were typically adjusted to recognize deflections exceeding $20 \%$ of the maximal spike amplitude occurring $>25 \mathrm{~ms}$ after the last spike. For the unipolar recordings during canine VF, the same criteria were applied to the first derivative of the electrogram. The peaks selected by the algorithm were
A Human AF
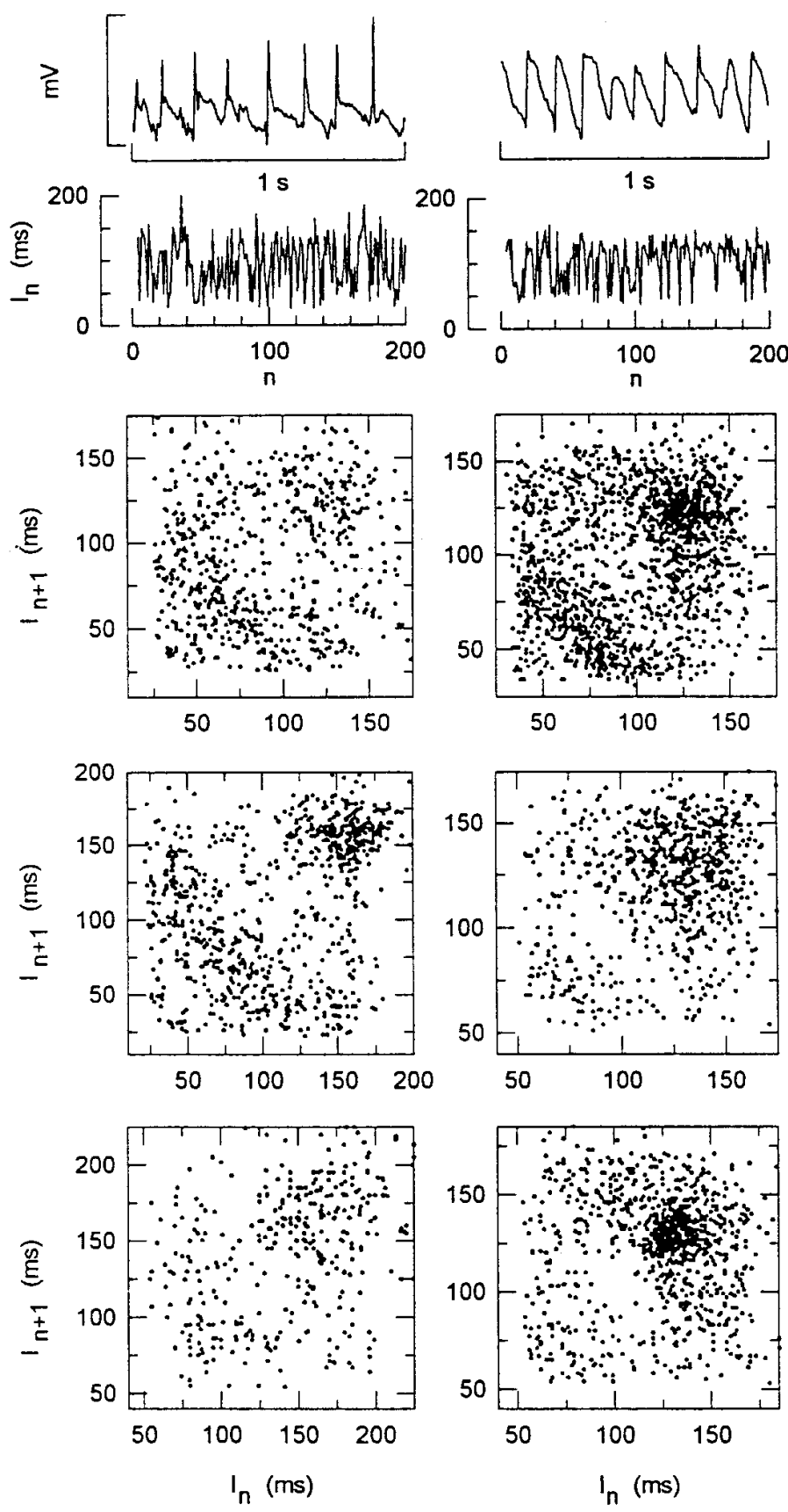

C RV Epicardium
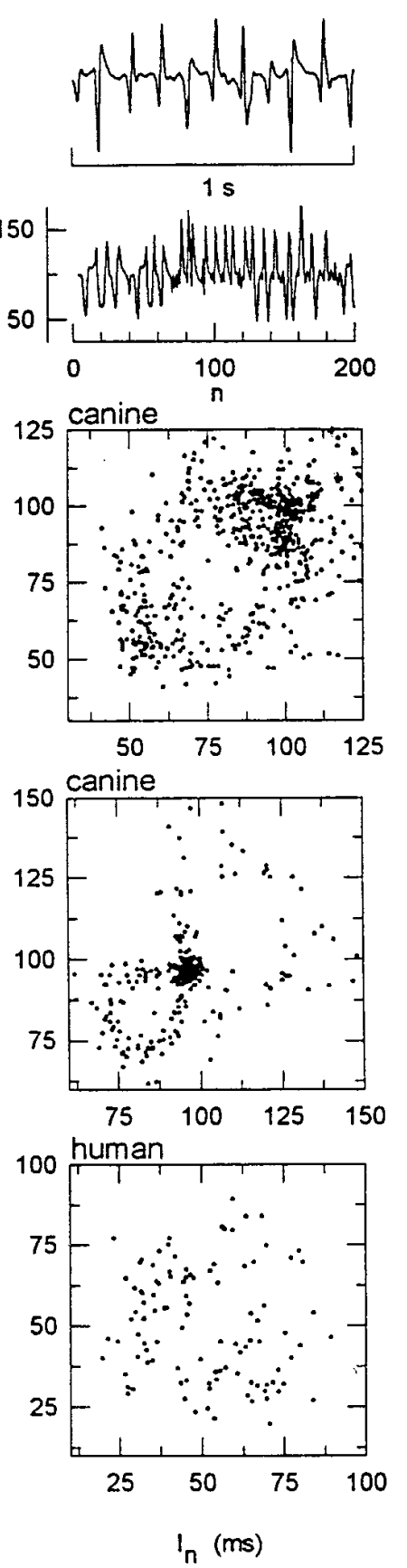

Figure 1. Intracardiac electrical activity during $(A)$ human atrial fibrillation, $(B)$ canine ventricular fibrillation, and $(C)$ a fibrillationlike state in sheets of isolated canine or human right ventricular epicardium. Top row: Brief extract of local intracardiac electrograms. Second row: series of interactivation intervals $\left(I_{n}\right)$ measured from the electrograms, illustrating the irregular patterns characteristic of fibrillation. Third row: Poincaré interval plots of $I_{n+1}$ against $I_{n}$, for the full data sets from which the upper rows are extracts. Fourth and fifth rows: Poincaré plots of $I_{n+1}$ against $I_{n}$, in $(A)$ two additional human patients with chronic atrial fibrillation, $(B)$ two additional canine hearts, and $(C)$ two additional epicardial sheet preparations, one canine (fourth row) and one human (fifth row). Note that in all three fibrillation models, the shortest interactivation intervals were in the range of $30-50 \mathrm{~ms}$. This is less than estimates of the refractory period during either atrial or ventricular fibrillation $(65,66)$, suggesting that the activations creating these short intervals result from less than fully developed action potentials or electrotonic events. These spikes may represent double potentials, which occur when the recording site is near the core of functional block of a reentrant wavefront (67), or when a wavefront generated by a second reentrant pathway collides nearby, producing an electrotonic depolarization at the recording site (66). We did not attempt to exclude electrotonic depolarizations, since they influence local excitability and hence propagation. 
visually inspected at multiple randomly chosen sections to confirm accuracy.

Poincaré plots were constructed from interactivation intervals of the electrograms by plotting the $\mathrm{n}^{\text {th }}$ interval against the $(\mathrm{n}+1)^{\mathrm{st}}$.

Computer simulation. The computer model consisted of an isotropic $128 \times 128$ lattice of excitable elements (a cell model of the cardiac action potential) coupled resistively, representing a rectangular patch of cardiac tissue $4 \mathrm{~cm}$ on a side. Two types of cell models were used: in some simulations, a modification of the Van Capelle/Durrer two-variable model (15), and in others, a three-variable simplification of the Luo/Rudy model (16). The parameters of these elements were adjusted to produce physiologically realistic values of: action potential duration (APD) $200 \mathrm{~ms}$, maximal rate of rise of the action potential upstroke $(\mathrm{dV} / \mathrm{dt})_{\max } 340 \mathrm{~V} / \mathrm{s}$, rectilinear conduction velocity $40 \mathrm{~cm} / \mathrm{s}$, and restitution properties of APD and $(\mathrm{dV} / \mathrm{dt})_{\max }$. The two-variable model does not reproduce the restitution properties of $(\mathrm{dV} / \mathrm{dt})_{\max }$ while the three-variable model does. The explicit numerical integration algorithm for the two-variable model was a forward Euler with a time step of $0.001 \mathrm{~ms}$, and for the three-variable model, an AshourHanna method with time step $0.03 \mathrm{~ms}$. The space step and the diffusion coefficient were the same for both models, equaling $0.03 \mathrm{~cm}$ and $1.2 \mathrm{~cm}^{2} / \mathrm{s}$, respectively. Simulations were performed on a massively parallel computer (model CM-2; Thinking Machines, Inc., Cambridge, MA).

\section{Results}

Poincaré plots. In contrast to random behavior, deterministic behavior means that the present state of a system is determined by its previous states. A simple test for such a relationship is a Poincaré plot, in which each successive value of a system variable is plotted against its previous value. For a purely random system, the distribution of points on a Poincare plot is formless, whereas for a system with significant nonrandom elements, the points often form a distinct structure. We constructed Poincaré plots of interactivation intervals (joint interval histograms of $I_{n}$ vs. $I_{n+1}$ ) during the three types of fibrillation. These intervals were measured in extracellular electrograms recorded directly at the myocardial surface. Electrograms exhibit a spike each time a wave of membrane depolarization passes the electrode (Fig. 1, first row); intervals between spikes are therefore a dynamic measure of local activity. During atrial or ventricular tachycardia, interactivation intervals are nearly constant, but during fibrillation they become highly irregular (Fig. 1, second row). In all three types of fibrillation, the Poincaré plots formed a distinctive pattern: a ringlike structure consisting of a relatively empty, roughly circular
A

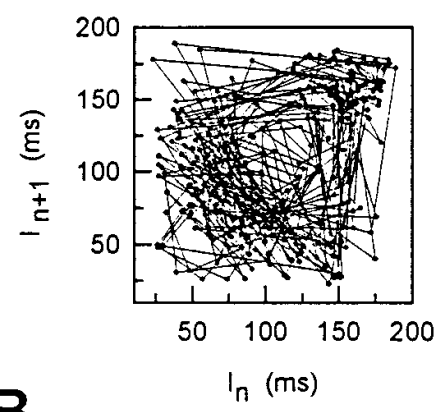

B
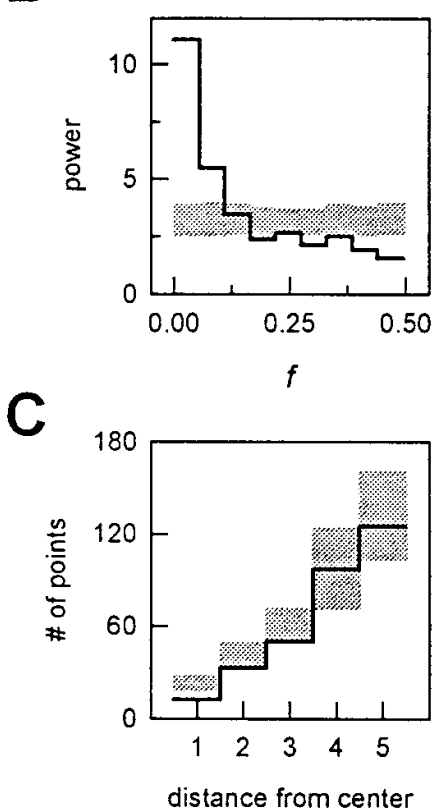

(arb. units)
Canine V Fib
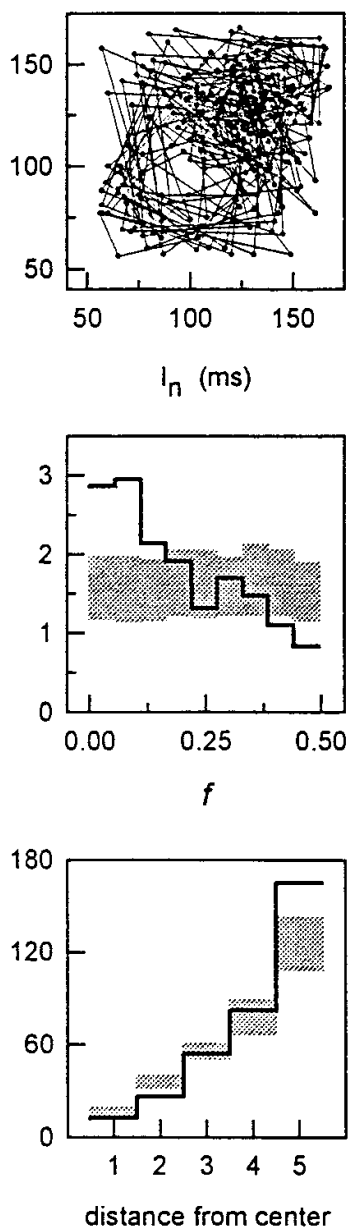

(arb. units)

\section{Canine RV Epicardial Sheet}
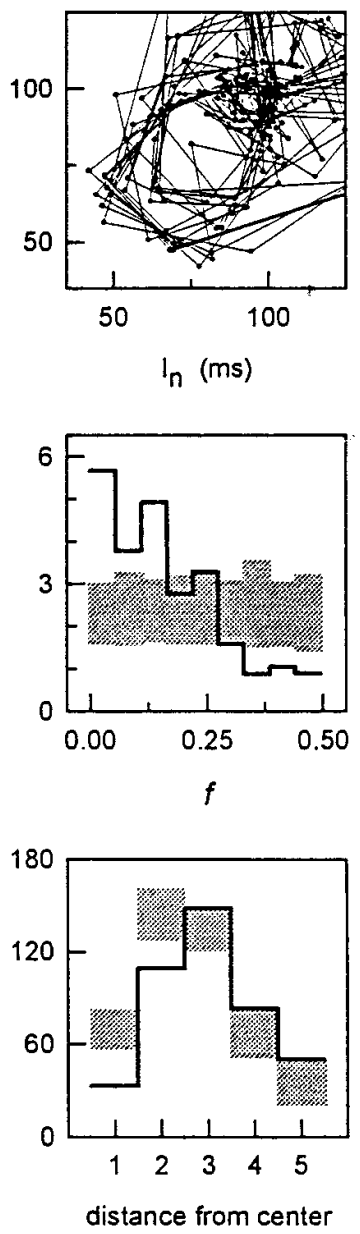

(arb. units)
Figure 2. A. Poincaré plots of interactivation intervals (from the examples in Fig. 1) in which lines have been drawn connecting successive points. Note that the lines tend to march around the perimeter rather than cross through the center of the ring-like structure, which implies a low-frequency modulation (20). B. Fourier spectra of interactivation intervals from the same data sets, demonstrating the lowfrequency modulation as a statistically significant peak in the lowest frequency (20). $C$. Histograms demonstrating the presence of a hole in the center of the Poincaré plots in $A$. In $B$ and $C$, the heavy black lines represent the actual data, while the shaded gray regions are the $99 \%$ confidence intervals for the same data in randomized order. Thus any part of the black line outside of the gray area is significant with $P<0.01$. 

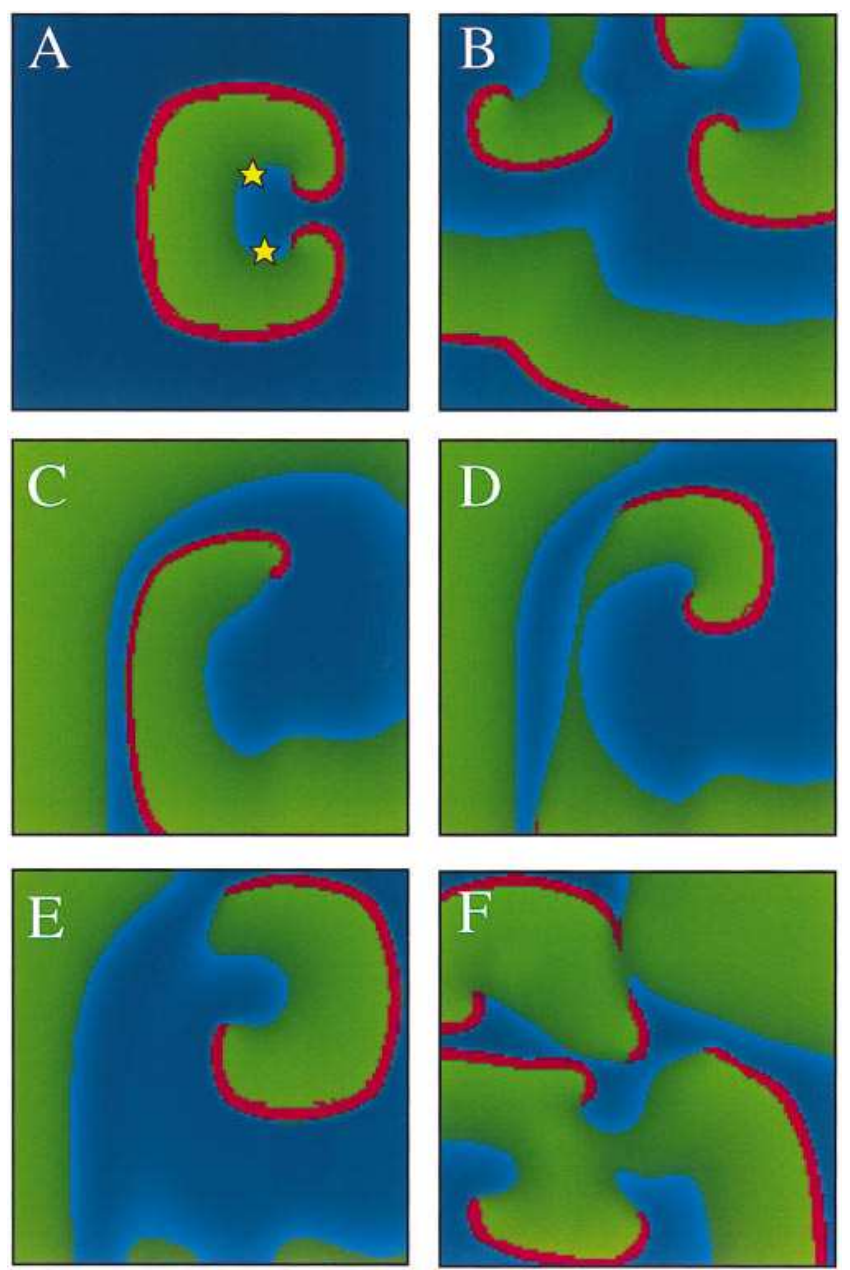

Figure 3. Spiral wave breakup leading to a fibrillation-like state in the computer simulation. $A-B$. Pacing-induced breakup. $A$ shows a pair of spiral waves shortly after their initiation by a $10 \times$ threshold premature pacing stimulus, delivered in the wake of a prior stimulated excitation wave. Two additional pacing stimuli (sites of delivery indicated by stars) broke up the two spiral waves into multiple meandering spiral waves $(B) . C-F$. Spontaneous spiral wave breakup. $C$ shows a single spiral wave immediately after its induction by a premature pacing stimulus, as in $A$. Note the variable wavelength (thickness of the red + green regions between the activation wavefront and waveback, equal to the product of the action potential duration and conduction velocity) along the arm of the spiral wave. In $D$, wave thickness has varied so much that the wave is about to break into two distinct pieces. In $E$, the original spiral wave and one of the broken ends form the cores of two counter-rotating spirals. The other broken end has migrated to the bottom edge and extinguished. After several repetitions of this scenario, the tissue reaches a fibrillation-like state in $F$. Color code represents $\mathrm{dV} / \mathrm{dt}$, with red marking the action potential upstroke, green the plateau, light blue the rapid repolarization phase, and dark blue, fully repolarized tissue. In the spontaneous breakup shown in $C-F$, the slope of the APD restitution curve is steeper than in $A-B$.

core region, surrounded by denser regions (Fig. 1, third through fifth rows). In a total of 19 different epochs of AF analyzed in five patients, 15 of the Poincaré plots showed visually clear ring-like structures, present in at least one epoch in each patient. In canine VF, ring-like structure was observed in 20 of 24 Poincaré plots constructed from 12 episodes of VF in the six canine hearts. In the fibrillation-like state of the ventricular epicardial sheet preparation (Fig. $1 C$ ), Poincaré plots of interactivation intervals showed ring-like structures in all three cases. The central hole in each ring-like structure was confirmed by spatial statistics (Fig. $2 C$ ), adapting a standard test (17), namely that if a hole exists, then few points will lie at short radial distances from its center. We chose a center within each ring in the data (some data sets had two rings), calculated the distance from the center to each of the data points (for two centers, we chose the smaller distance), and tallied those distances into a 20-bin histogram. We then shuffled the intervals, reconstructed the Poincaré interval plot and calculated a new histogram of distances. After 100 shuffles, every data set showed at least one bin for short radii whose tally was less than all 100 of the histograms of the shuffled data; thus, in all cases, there is a statistically significant hole, with $P<0.01$.

In the computer model (with the two-variable cell model), using the same stimulus protocol that induced reentry in the experimental preparations $(12,13)$, single or paired spiral waves were readily induced (Fig. $3 \mathrm{~A}$ ). Extra stimuli created additional, meandering spiral waves, simulating a fibrillationlike state (Fig. $3 \mathrm{~B}$ ). In addition, using the three variable cell model, the appropriate choice of electrophysiological parameter values (see below) resulted in spiral wave breakup, spontaneously producing a fibrillation-like state (Fig. $3 C-F$ ). Fig. 4 A-B show intracellular membrane potential $(A)$ and its first derivative $(B)$ at a local site during the simulated fibrillation as in Fig. $3 \mathrm{~B}$. A series of interactivation intervals from the full record is shown in Fig. $4 C$, its Poincaré plot in Fig. $4 E$ and a Poincaré map from a different simulation in Fig. 4 I. As with the experimental data, the Poincaré plots show clear ring-like structures, confirmed by the same spatial statistics. Thus, the computer model simulated key features observed during fibrillation in the various experimental models. In addition to the analyses of the discrete series of intervals, we also used timedelay embedding techniques $(18,19)$ to construct a continuous three-dimensional trajectory of the intracellular membrane potential at the same local site. A Poincaré section of this trajectory is illustrated in Fig. $4 G$, and shows a partial ring-like structure, reflecting a broken torus (see below). Thus, equivalent results were obtained using either the discrete set of interactivation intervals or a continuous flow.

Tests for quasiperiodicity. The ring-like structures in the Poincaré plots above suggest chaos that has arisen from quasiperiodicity $(18,19)$. A system's behavior is said to be quasiperiodic if it displays several independent frequencies, for example an oscillation at one frequency that is amplitude-modulated at another frequency. When a quasiperiodic system becomes chaotic, the quasiperiodic frequencies often remain detectable in the chaotic regime $(18,19)$. One positive indication of quasiperiodicity in our fibrillation data is that lines connecting successive points of the Poincaré plot rarely cross the central hole; instead, they march around its perimeter, indicating low-frequency modulation of the intervals (Fig. $2 A$ ). This quasiperiodicity was confirmed statistically by calculating Fourier spectra of the interval sequences (Fig. $2 B ; 20$ ). The power density was calculated between 0 to 0.5 cycles/activation spike, binned into nine equal ranges. The statistical significance of the spectra (heavy lines) was assessed by comparing it with 100 spectra calculated for the same set of intervals, shuffled into pseudo random order. The $1^{\text {st }}$ and $99^{\text {th }}$ percentiles of the values in each frequency range for the shuffled data are indicated by the gray 
A

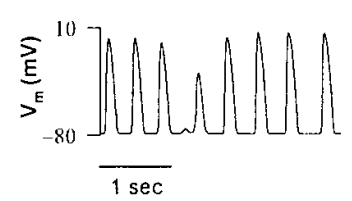

B

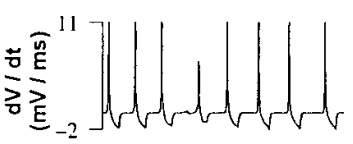

C

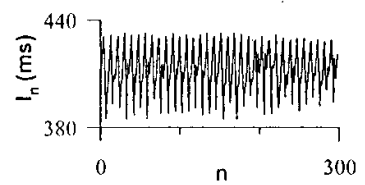

D

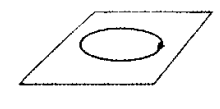



E
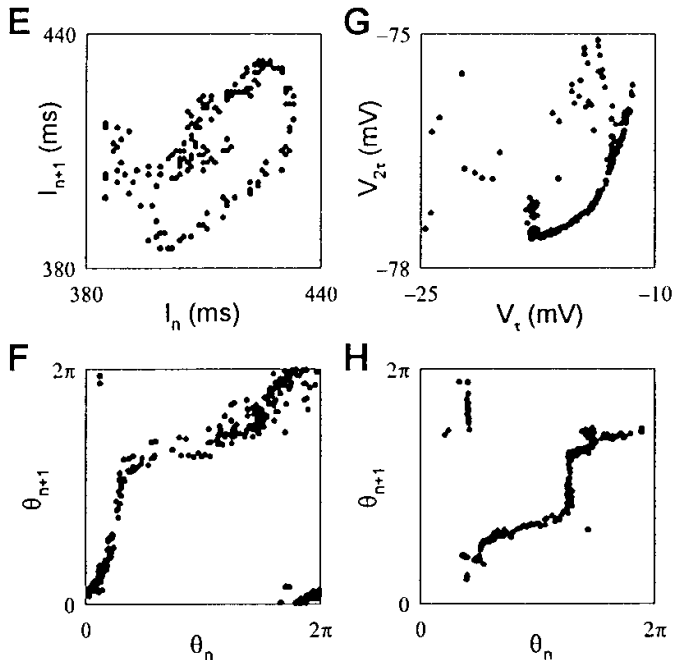
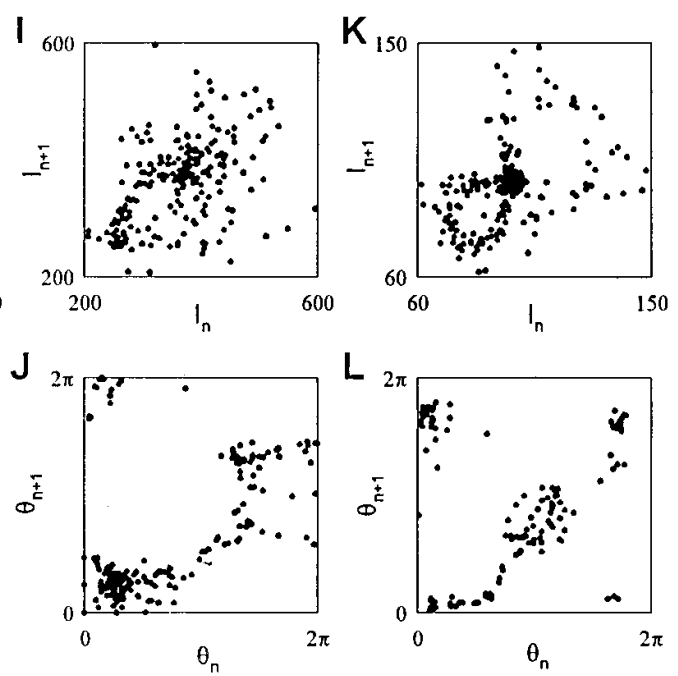



Figure 4. Torus breakdown. $A-B$. Intracellular potential $(A)$ and its first derivative $(B)$ recorded at a local site during the fibrillation-like state of the computer simulation, with multiple meandering spiral waves. The first derivative simulates an extracellular electrogram, as recorded in Fig. 1. C. A series of successive interactivation intervals during the fibrillation-like state. $D$. Schematic diagram explaining origin of ring-like structures in Poincaré sections of quasiperiodic behavior (18, 19,38). $E$ and $I$. Poincaré plots of interactivation intervals during two simulations of fibrillation. Note the similarities to the ring-like structure in the Poincare plots in Fig. 1. $F$ and $J$. Plots of $\theta_{\mathrm{n}} \mathrm{vs}$. $\theta_{\mathrm{n}+1}$ for the interactivation interval plots in $E$ and $I$. The $\theta_{\mathrm{n}} / \theta_{\mathrm{n}+1}$ plots are noninvertible functions, indicative of quasiperiodic chaos (see text). $G$. Poincaré section of the continuous three-dimensional trajectory of intracel-

lular membrane potential (delay embedding with $\tau=100 \mathrm{~ms}$ ) from the data set represented in $A$. $H$. Plot of $\theta_{\mathrm{n}}$ vs. $\theta_{\mathrm{n}+1}$ for the Poincaré section in $G$. As in $F, J$, and $L$, the relationship between $\theta_{\mathrm{n}}$ vs. $\theta_{\mathrm{n}+1}$ is noninvertible. $K$ and $L$. Poincaré plot of interactivation intervals $(K)$ and $\theta_{\mathrm{n}}$ vs. $\theta_{\mathrm{n}+1}$

$(L)$ for canine epicardial sheet preparation. The polar angle plot also suggests a noninvertible relationship between $\theta_{\mathrm{n}}$ vs. $\theta_{\mathrm{n}+1}$.

shading. In all cases, statistically significant power appeared in the low-frequency band around 0.2 cycles per activation, representing modulation over $\sim 5$ intervals.

Circle maps. Several pathways from quasiperiodicity to chaos have been described, such as torus breakdown (21) and torus doubling. The main diagnostic criterion for the torus breakdown pathway is the way in which the state point progresses around the ring-like structure in the Poincaré plot (Fig. $4 D$ ). Choosing an origin within the ring, let $\theta_{\mathrm{n}}$ be the polar angle that the $\mathrm{n}^{\text {th }}$ point makes with that origin. In quasiperiodicity, the plot of $\theta_{n}$ against $\theta_{n+1}$ will be a monotonically increasing function (modulo $2 \pi$ ), reflecting the steady precession of the state point around the ring. In the transition to chaos, this function (sometimes called a circle map) becomes nonmonotonic. Finally, as the chaos develops further, the $\theta_{n} / \theta_{n+1}$ plot ceases to be a function at all, and becomes a cloud of points. Our computer simulations of fibrillation, as well as the fibrillation-like state in the epicardial sheet preparation, showed this sequence: Fig. $4, F$ and $J$ show the plots of $\theta_{\mathrm{n}}$ vs. $\theta_{\mathrm{n}+1}$ constructed from the Poincaré maps of interactivation intervals in Fig. 4, $E$ and $I . \theta_{\mathrm{n}+1}$ is approximately a single-valued function of $\theta_{\mathrm{n}}$, but the nearly horizontal segment $\left(\pi / 2<\theta_{\mathrm{n}}<\pi\right)$ makes the relationship between $\theta_{\mathrm{n}}$ and $\theta_{\mathrm{n}+1}$ nonmonotonic and hence noninvertible (i.e., there is no longer a unique value of $\theta_{\mathrm{n}}$ for each $\left.\theta_{\mathrm{n}+1}\right)$. The plot of $\theta_{\mathrm{n}}$ versus $\theta_{\mathrm{n}+1}$ constructed from the Poincaré section of the continuous three-dimensional tra- jectory of membrane potential (Fig. $4 G$ ) was also noninvertible (Fig. $4 \mathrm{H}$ ). In the Poincaré plot from the epicardial sheet tissue (Fig. $4 K$ ), the $\theta_{\mathrm{n}}$ vs. $\theta_{\mathrm{n}+1}$ plot was roughly one-dimensional and showed regions of noninvertibility (Fig. $4 \mathrm{~L}$ ), thus supporting the diagnosis of chaos that arose from a quasi-periodic regime, via torus breakdown. In our data from human AF and canine VF, however, phase angle relationships were too thickened to determine whether $\theta_{\mathrm{n}}$ and $\theta_{\mathrm{n}+1}$ fell on a noninvertible curve. In these cases, the findings are consistent with, but not diagnostic of, fully developed chaos.

Lyapunov exponents. One defining feature of chaos is exponential divergence of nearby trajectories. To determine whether this property could be detected in the fibrillation data, we attempted to estimate Lyapunov characteristic exponents in an appropriate delay-coordinate embedding (22). This requires rejecting false nearest neighbors, which arise when the embedding dimension is too low $(23,24)$. In our computer simulations, the percentage of false nearest neighbors dropped below $2 \%$ at an embedding dimension of four. This was low enough to permit estimating the largest Lyapunov exponent, which was positive (range 0.08-0.14), indicating chaos. In the biological fibrillation data, however, the proportion of false nearest neighbors was too high, at any plausible embedding dimension, to allow reliable estimation of Lyapunov exponents. We suspected that this was due to the limited precision of the biological data, so we truncated the computer data to that pre- 


\section{A. Canine RV Epicardium}

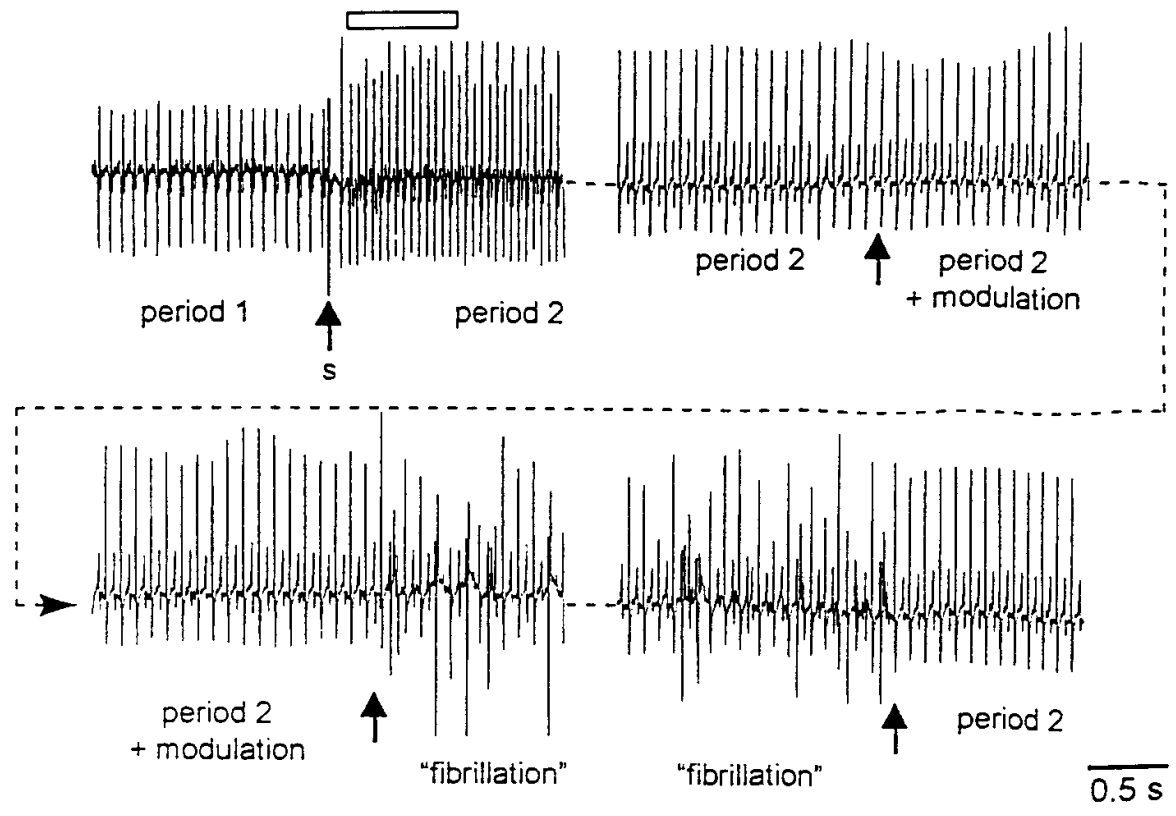

\section{B. Human RV Epicardium}
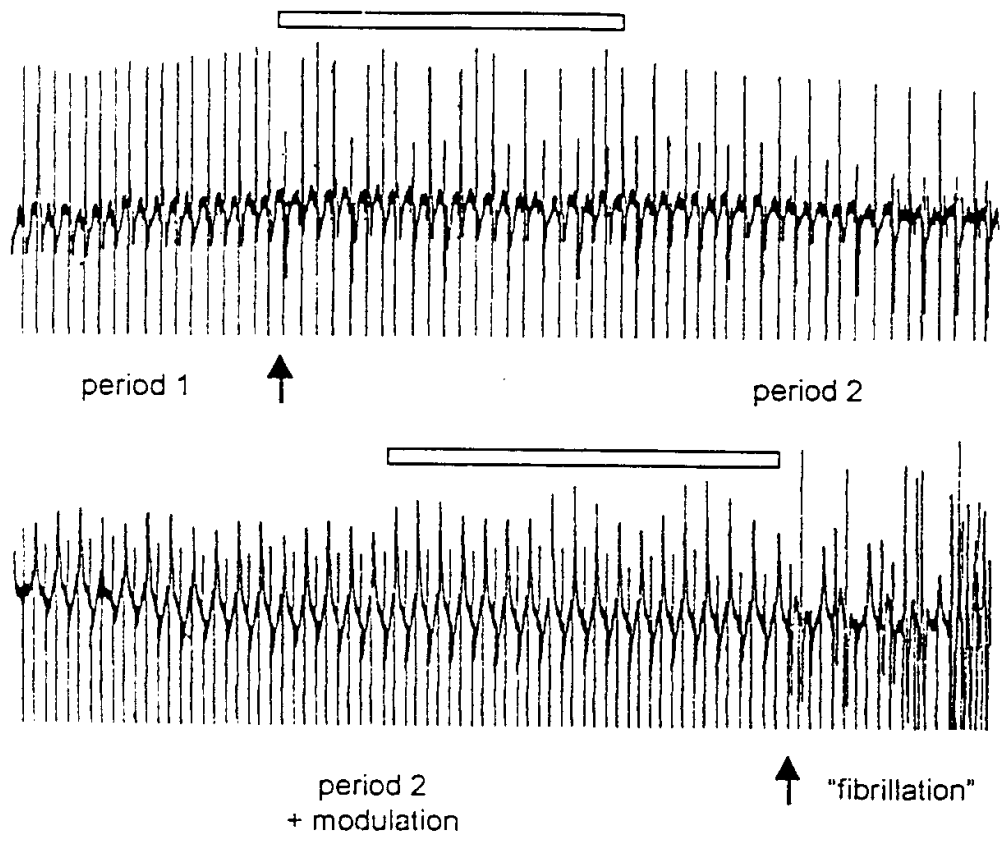

Figure 5. Transition from periodic state (spiral-wave tachycardia) to a fibrillationlike state in epicardial sheets prepared from canine $(A)$ and human right ventricle (B). A. After induction of sustained reentry in the presence of cromakalim $(10 \mu \mathrm{M})$, the extracellular electrogram showed stable period-1 behavior (beginning of trace), until an external pacing stimulus (at $s$ ) shortened the cycle length. After an initial transient instability (indicated by overlying box), the accelerated beats settled into an alternating (period-2) pattern in both the amplitude and period (52 $\mathrm{ms}$ alternating with $45 \mathrm{~ms}$ ) of the spikes. The period-2 pattern continued for several minutes, evolving gradually with accentuation in the difference between amplitudes of the two spikes (right upper trace). However, at the second arrow (right upper trace), a slow modulation of the amplitude and period of the large spike began spontaneously (period-2 + modulation), leading to a sudden transition to highly aperiodic fibrillationlike behavior (arrow, left lower trace). The fibrillation-like state persisted for several seconds before spontaneously reverting to the period-2 behavior (arrow, right lower trace). B. A similar sequence recorded from a human right ventricular epicardial sheet preparation in the presence of $10 \mu \mathrm{M}$ cromakalim. In this example, the initial transition (arrow, upper trace) occurred spontaneously, taking the period-1 reentrant tachycardia (cycle length $130 \mathrm{~ms}$ ) into period-2 behavior through a transient unsettled period (indicated by the overlying box). The period- 2 behavior then became progressively modulated (lower trace), reflected by a growing oscillation in the amplitude of the upward spikes (indicated by overlying box), leading to a sudden transition to the fibrillation-like state (arrow). cision $(\sim 0.5 \mathrm{~ms})$. This truncated computer data, like the biological data, failed to meet the criterion of few enough false nearest neighbors, and hence, its Lyapunov exponents could not be estimated. This reveals a difficulty in applying traditional numerical measures, developed for diagnosing low dimensional chaos, to higher dimensional biological systems in which data can be obtained with only limited accuracy (24-27).

Direct evidence of quasiperiodicity preceding fibrillation. Direct evidence linking quasiperiodicity and fibrillation came from the epicardial sheet preparations, in which we observed the onset of fibrillation in several instances (Fig. 5). In two cases, one from canine (Fig. $5 A$ ) and one from human (Fig. 5
$B$ ) ventricular tissue, fibrillation was preceded by a long epoch of periodic behavior (period-2 or alternans), which then became unstable for a few seconds. The instability took the form of growing oscillatory modulations in both amplitude and period, followed by an abrupt transition to fibrillation. Because this modulated behavior is quasiperiodicity, the tissue has indeed evolved from regularity, through quasiperiodicity, to chaotic fibrillation.

Activation maps. In the examples in Fig. 5, detailed spatial activation maps were not obtained during the transition to fibrillation. Fig. 6 shows another experiment in which the activation sequence was mapped for $3.5 \mathrm{~s}$ during the transition from 

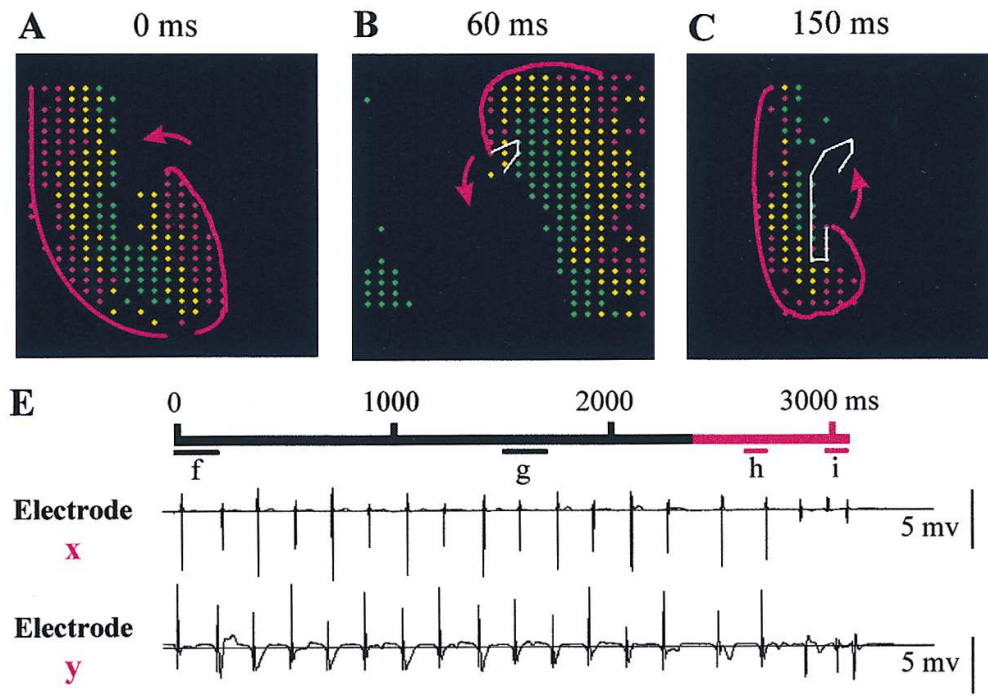

Electrode



G

H

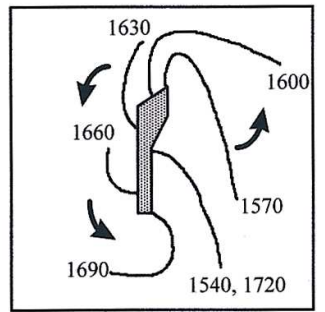

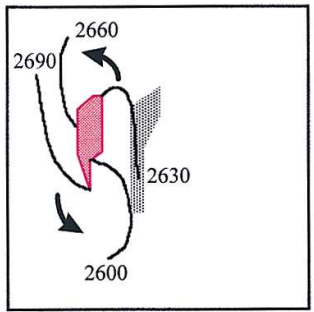

I

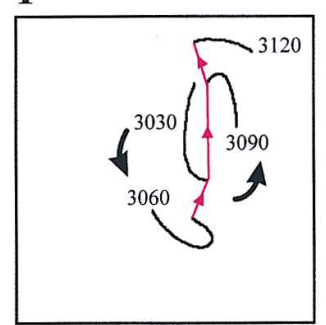

D $\quad 180 \mathrm{~ms}$



F

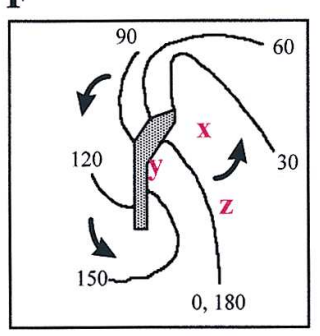

J

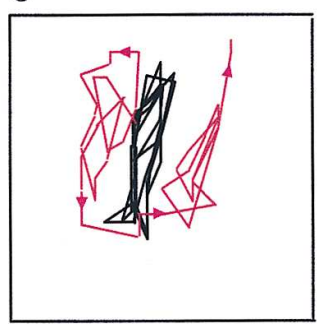

Figure 6. Activation mapping during the transition to a fibrillation-like state in a canine RV epicardial sheet preparation, measuring approximately $25 \mathrm{~mm} \times 25$ mm. $A-D$. Snapshots of the position of activation wavefront at indicated intervals during one rotation of a spiral wave (epoch $f$ in $E$ ). Most recently activated sites are indicated by red, and the color changes from red to yellow to green to black every $10 \mathrm{~ms}$. Red line shows the leading edge of the spiral wave; white lines trace the path of the tip of the spiral wave. The mapping was performed using an array of 509 closely spaced bipolar electrodes $(12,13)$. E. Representative electrograms from three sites (x, y, and $\mathrm{z}$ in $F$ ) during the $3.5 \mathrm{~s}$ duration of mapping. $F$. Isochronal activation map for the rotation of the spiral wave (epoch $f$ in $E$ ) illustrated in $A-D$. The core of the spiral wave is the shaded area outlined by the white lines in $D$. G. Isochronal activation map of the spiral wave starting at $1,540 \mathrm{~ms}$

(epoch $g$ ) shows an almost identical pattern. $H$. At $\sim 2450 \mathrm{~ms}$, (epoch $h$ ) the spiral wave spontaneously began to meander rapidly; the isochronal activation map beginning at 2,600 ms shows that the core ( red) has shifted to the left. The position of the original core is indicated by the gray shaded area. $I$. The isochronal activation map during the final rotation of the spiral wave (epoch $i$ ) which extinguishes when it runs into the upper border of the sheet (the path of the spiral tip is shown in red). $J$. The trajectory of the tip of the spiral wave (every $20 \mathrm{~ms}$ ) during the transition from the stationary (black lines) to hypermeander (red lines) of the spiral wave. Note that during the stationary phase, slight but definite meander of the tip trajectory was present (i.e., the black lines do not superimpose exactly).

a stable spiral wave to a brief nonsustained fibrillation-like state. During the initial $2.3 \mathrm{~s}$, the core of the spiral wave was relatively stable, showing only a slight meander. At a recording site distant from the core (electrode $\mathrm{z}$ ), activations were monomorphic. Closer to the core, however (electrode $\mathrm{x}$ ), an alternating pattern was observed; at the margin of the core (electrode y), the alternans was strongly modulated. We attribute the latter modulation to the definite though slight meandering motion of the core during successive rotations. We hypothesize that the multiple coupled oscillations at the core destabilized the spiral wave because at $2.3 \mathrm{~s}$, the spiral wave suddenly began to meander violently, with the tip of the spiral wave tracing the trajectory indicated in red in Fig. 6J. This hypermeander (28) led to termination of the spiral wave after only six additional rotations; during this stage the electrograms at all three sites became highly disordered, similar to those observed during the fibrillation-like state in the examples in Fig. 5. Hypermeander of a single spiral wave has recently been shown in the isolated rabbit heart to produce the electrocardiographic pattern of fibrillation (29). Subsequent breakup of a single meandering spiral wave into multiple spiral waves may also occur in sustained fibrillation. In the preparations in Fig. $5 \mathrm{~B}$, crude activation mapping during the fibrillation-like state showed the presence of multiple circulating wavefronts (data not shown). These experimental mapping results further support the relevance of spiral-wave hypermeander and breakup in the computer simulation to genuine cardiac fibrillation.

Spiral breakup. Unstable quasiperiodicity is also the cause of spiral breakup leading to the fibrillation-like state in the computer simulation. In Fig. $3 C-F$, the wavelength of the excitation wave (APD $\times$ conduction velocity) is shown by the thickness of the red plus green areas. Shortly after initiation of the spiral wave, the wavelength shows an oscillatory scalloping (i.e., a quasiperiodic modulation of wavelength), as one moves outward along the arm of the spiral wave (Fig. $3 \mathrm{C}$ ). This occurs because the fundamental period of the spiral wave is too short to allow full recovery of tissue in the path of the next wavefront, so that $(\mathrm{dV} / \mathrm{dt})_{\max }$ and APD recover incompletely, as determined by their restitution properties. As the spiral arm rotates, this modulation of wavelength intensifies until finally, at one location, the APD (and hence the wavelength) has become too short to successfully propagate (Fig. $3 \mathrm{D}$ ). This 
pinches off the arm of the spiral wave, creating two new ends, which form the tips of two new spiral waves (Fig. $3 E$ ). This breakup process repeats itself, producing the multiple, often fractionated spiral waves, characteristic of the fully developed fibrillation-like state (Fig. $3 F ; 30$ ).

\section{Discussion}

The quasiperiodic transition to chaos. The idea that multifrequency quasiperiodicity is inherently unstable, and will degenerate into chaos, was first suggested by Ruelle and Takens in 1971, in application to fluid turbulence. Classically, turbulence was thought to result from the accumulation of many independent oscillatory motions (31). This was shown to be mathematically impossible by Ruelle, Takens, and Newhouse $(32,33)$, who proved that a system containing three or more coupled oscillations is unstable. Their predictions were confirmed experimentally by the elegant fluid dynamics studies of Swinney and others (34-36), and have been extended to other systems such as electronic materials (37).

The quasiperiodic scenario explains the origin of the ringlike structures seen in the Poincaré plots. Consider the state of a quasiperiodic system consisting of two coupled oscillations. The evolving state is represented by a point moving in a trajectory on the surface of the doughnut-shaped 2-torus, $\mathrm{T}^{2}$, whose two cyclic coordinates represent the two oscillations $(18,19$, 38; Fig. $4 \mathrm{D}$ ). Suppose we sample this trajectory by sectioning the torus with a plane, $\mathrm{P}$, transverse to one of the cyclic coordinates. If the behavior is periodic, the trajectory will intersect the sectioning plane at a discrete set of points. But if the behavior is quasiperiodic, the trajectory intersects at an infinite set of points that precess around a simple closed curve. If the quasiperiodicity progresses to chaos, the ring-like structures will thicken and/or break $(18,19)$. Thus, ring-like structures around which the state point precesses (see below), such as observed in our fibrillation data, suggest chaos that has arisen from quasiperiodicity. Similar ring-like structures have been observed in other physical systems undergoing transitions from quasiperiodicity to chaos, such as the transition to turbulence in fluids (34-36) and to chaos in electronic materials (37). In addition, mathematical models, which, like cardiac conduction, are governed by reaction-diffusion equations in excitable media, have been shown to undergo transitions to spatio-temporal chaos via a quasiperiodic route $(39,40)$. In each of these cases, thickened and sometimes broken ring-like structures were observed in Poincaré plots, like those observed in our fibrillation data.

Since Ruelle and Taken's original papers $(32,33)$, additional pathways from quasiperiodicity to chaos have been described, such as torus breakdown and torus doubling. In fluid turbulence, electronic materials and spatiotemporal chaos in excitable media, the quasiperiodic transition to chaos often takes place via a route that involves the chaotic destruction of the torus (torus breakdown). This has a characteristic appearance in the circle map, which records the progression of the state point around the torus. If the state point is truly staying on the surface of the torus, then the function relating the current position to the previous must be constantly increasing, i.e., monotonic, reflecting the progression around the circle. If, however, the point appeared to move backward on the circle, i.e., a nonmonotonic circle map, that would indicate that the state point is no longer in a deterministic flow on the surface of a torus $(18,19)$. Such nonmonotonic (or noninvertible) circle maps are found in each of the examples in the literature of the torus breakdown scenario $(34-37,39,40)$.

In our computer simulations, the evidence for a quasiperiodic transition to chaos via the torus breakdown route is decisive: ring-like structure in the Poincaré plot, low-frequency modulation, a transition to a noninvertible circle map, and positive Lyapunov exponent. The biological preparations also displayed Poincaré structure, low frequency period and amplitude modulation, and, in the epicardial sheet preparation, a nonmonotonic, noninvertible circle map; in addition, they contained transitions to fibrillation, which were immediately preceded by short segments of unstable quasiperiodicity. Thus, our findings provide the first experimental validation of this scenario in a biological setting, and suggest that quasiperiodic transitions may underlie spatiotemporal chaos in other excitable media which generate spiral waves, including chemical reactions (41) and $\mathrm{Ca}^{2+}$ waves $(42,43)$.

Identifying the quasiperiodic oscillations. The biological results and computer simulations provide insight into the various oscillations and modulations that constitute the quasiperiodic scenario. The primary oscillation is a spiral wave, a rotating wave of excitation that repeatedly reenters each region in its path $(11,44-47)$. The second oscillation is alternans, which arises because the cells in the path of the rotating spiral are being reexcited before they have completely recovered from the previous excitation. Alternans due to rapid periodic forcing of heart cells is well known (48). It is generally attributed to the cellular property of restitution, which relates each action potential duration (APD) or the maximal action potential upstroke velocity, $(\mathrm{dV} / \mathrm{dt})_{\max }$, to the previous diastolic interval (49), and hence to the driving rate. The third and fourth oscillations in the quasiperiodic scenario are the period and amplitude modulations that immediately precede and precipitate the transition to chaos. They correspond to the onset of meander of the primary spiral wave, which is mathematically a transition to quasiperiodicity, and has been shown to cause quasiperiodic modulations of period and amplitude in heart tissue $(29,50,51)$. Theoretical studies of cardiac wave propagation in one-dimensional rings of tissue (52) have attributed the onset of quasiperiodicity to APD restitution and the restitution of $(\mathrm{dV} / \mathrm{dt})_{\max }$, and similar quasiperiodic modulations have been observed in rings of cardiac tissue (53).

Limitations. True VF in vivo is more complicated than in the two experimental ventricular preparations we have studied. In one preparation, the $\mathrm{K}^{+}$channel opener cromakalim was required to induce VF; in both preparations, VF was initiated by external electrical stimulation rather than spontaneously, and destabilizing factors such as progressive ischemia and increased sympathetic tone were absent. Avoiding these nonstationary factors was essential for our methods. More work will be necessary to assess the dynamic consequences of these nonstationary factors, which are highly relevant to clinical VF. Nonstationarity may have accounted for negative results in previous studies of chaos in VF, which have been mixed (54-56). Two negative studies used preparations that were hemodynamically unstable during fibrillation, and one used chest-surface EKGs, which are averages over the spatially disorganized activity, rather than local measures. The direct relevance of our findings to human disease is supported by the results in chronic human AF, in which the same hallmarks of spatiotemporal chaos were observed as in our VF models. 
Another limitation is that the computer simulations were simplified, and did not incorporate any anatomically based spatial inhomogeneities (e.g., anisotropy) or randomness in the electrophysiological properties of the tissue (e.g., dispersion of refractoriness; 2 ). On the other hand, the simulations demonstrate that spiral wave breakup causing fibrillation by the torus breakdown scenario can occur in the absence of these factors. Although significant inhomogeneity exists in all real hearts and may enhance the tendency of the heart to fibrillate, it is not necessary as a prerequisite for fibrillation to develop by the torus breakdown scenario.

Therapeutic implications. A quasiperiodic route to chaos, as a cause of cardiac fibrillation, has specific therapeutic implications: strategies aimed at reducing or decoupling the oscillations may help prevent fibrillation. For example, clinical and experimental studies have shown that electrocardiographic $\mathrm{T}$ wave alternans is a precursor of ventricular arrhythmias, including VF (57-62). We think it is likely that the $\mathrm{R}$ wave amplitude and period alternans preceding fibrillation in our local electrograms was accompanied by alternans in repolarization, given the typical overlap between $(\mathrm{dV} / \mathrm{dt})_{\max }$ and APD restitution curves in cardiac tissue. However, this point remains to be proven, since we had no direct measure of repolarization in these experiments. Nevertheless, our study strongly supports previous evidence (62) that, rather than being an epiphenomenon, alternans is causally important in the development of fibrillation. Therefore, pharmacologic or pacing methods (63) to prevent or reduce alternans should have an antifibrillatory effect. In particular, the deactivation kinetics of delayed rectifier potassium currents, recovery from inactivation of inward currents, and intracellular $\mathrm{Ca}^{2+}$ cycling have been shown to be important determinants of restitution characteristics and alternans in APD, and offer realistic targets for modification by antiarrhythmic drugs. For example, previous studies have shown that $\mathrm{Ca}^{2+}$ channel blockers inhibit both electrical $(\mathrm{T}$ wave) alternans and predisposition to ischemic $\operatorname{VF}(59,61)$. Pacing strategies based on chaos control theory have also been shown to be effective in a simpler cardiac arrhythmia (64), and may conceivably be adapted to fibrillation.

\section{Acknowledgments}

We thank Drs. William Stevenson, Leslie Saxon, Holly Middlekauff, Isaac Wiener, David Delurgio, and Paul Natterson for their help in the human data collection, and Scott Lamp, Dustan Hough, Avile McCullen, Meiling Yuan, Tan Duong, and Zhilin Qu for technical assistance. We are grateful to Reggie Brown, Henry Abarbanel, and Michael Rosenstein for helpful discussions on Lyapunov exponents. Our thanks to the Ralph M. Parsons Foundation and the ECHO Foundation.

This paper was supported by a National Institutes of Health (NIH) SCOR in Sudden Cardiac Death 1P50HL-52319, NIH grants 1RO1HL-44880 and 1R2946770, the Laubisch Fund and the Kawata Endowment. Dr. Chen is an Established Investigator of the American Heart Association.

\section{References} 196.

Lewis, T. 1911. The Mechanism of the Heartbeat. Shaw \& Sons, London.

2. Moe, G.K., W.C. Rheinboldt, and J.A. Abildskov. 1964. A computer model of atrial fibrillation. Am. Heart J. 67:200-220.

3. Allessie, M.A., W.J.E.P. Lammers, F.I.M. Bonke, and J. Hollen. 1985. Experimental evaluation of Moe's multiple wavelet hypothesis of atrial fibrilla- tion. In Cardiac Arrhythmias. D.P. Zipes and J. Jalife, editors. Grune \& Stratton, New York. 265-276.

4. Damle, R.S., N.M. Kanaan, N.S. Robinson, Y.Z. Ge, J.J. Goldberger, and A.H. Kadish. 1992. Spatial and temporal linking of epicardial activation directions during ventricular fibrillation in dogs. Evidence for underlying organization. Circulation. 86:1547-1558.

5. Bayly, P.V., E.E. Johnson, P.D. Wolf, H.S. Greenside, W.M. Smith, and R.E. Ideker. 1993. A quantitative measurement of spatial order in ventricular fibrillation. J. Cardiovasc. Electrophysiol. 4:533-546.

6. Witkowski, F.X., K.M. Kavanagh, P.A. Penkoske, R. Plonsey, M.L. Spano, W.L. Ditto, and D.T. Kaplan. 1995. Evidence for determinism in ventricular fibrillation. Phys. Rev. Lett. 75:1230-1233.

7. Guevara, M.R., L. Glass, and A. Shrier. 1981. Phase locking, period-doubling bifurcations, and irregular dynamics in periodically stimulated cardiac cells. Science (Wash. DC). 214:1350-1353.

8. Chialvo, D.R., R.F. Gilmour, and J. Jalife. 1990. Low dimensional chaos in cardiac tissue. Nature (Lond.). 343:653-657.

9. Savino, G.V., L. Romanelli, D.L. Gonzalez, O. Piro, and M.E. Valentinuzzi. 1989. Evidence for chaotic behavior in driven ventricles. Biophys. J. 56: 273-280.

10. Nwasokwa, O.N., and M.M. Bodenheimer. 1989. Analysis of myocardial isometric dynamics using parameters of a global model. Am. J. Physiol. 257: H1275-H1286.

11. Davidenko, J.M., A.V. Pertsov, J.R. Salomonsz, W. Baxter, and J. Jalife. 1992. Stationary and drifting spiral waves of excitation in isolated cardiac muscle. Nature (Lond.). 355:349-351.

12. Bonometti, C., C. Hwang, D. Hough, J.J. Lee, M.C. Fishbein, H.S. Karagueuzian, and P.S. Chen. 1995. Interaction between strong electrical stimulation and reentrant wavefronts in canine ventricular fibrillation. Circ. Res. 77: 407-416.

13. Chen, P.S., P.D. Wolf, E.G. Dixon, N.D. Danieley, D.W. Frazier, W.M Smith, and R.E. Ideker. 1988. Mechanism of ventricular vulnerability to single premature stimuli in open-chest dogs. Circ. Res. 62:1191-1209.

14. Rensma, P.L., M.A. Allessie, W.J.E.P. Lammers, F.I.M. Bonke, and M.J. Schalij. 1988. The length of the excitation wave as an index for the susceptibility to reentrant atrial arrhythmias. Circ. Res. 62:395-410.

15. Kogan, B.Y., W.J. Karplus, and M.G. Karpoukhin. 1996. The Van Capelle and Durer model of cardiac action potential generation: modifications and applications to spiral wave propagation. In Simulation in Medical Sciences. J.G. Anderson and M. Katzper, editors. Society for Computer Simulation, San Diego. 106-112.

16. Kogan, B.Y., W.J. Karplus, and M.G. Karpoukhin. 1995. The thirdorder action potential model for computer simulation of electrical wave propagation in cardiac tissue. In Computer Simulations in Biomedicine. H. Power and R.T. Hart, editors. Computational Mechanics Publishers, Boston. 147-154.

17. Ripley, B.D. 1981. Spatial Statistics. John Wiley \& Sons, New York. 152-158.

18. Hilborn, R.C. 1994. Chaos and Nonlinear Dynamics. Oxford University Press, New York. 252-294.

19. Nayfeh, A.H., and B. Balachandran. 1995. Applied Nonlinear Dynamics. John Wiley \& Sons, New York. 314-334.

20. Gilmour, R.F., M. Watanabe, and D.R. Chialvo. 1993. Low dimensional dynamics in cardiac tissues. Experiments and theory. In Chaos in Biology and Medicine. W.L. Ditto, editor. SPIE-The International Society for Optical Engineering, Bellingham, WA. 2-9.

21. Curry, J.H., and J.A. Yorke. 1978. A transition from Hopf bifurcation to chaos: computer experiments with maps on $\mathrm{R}^{2}$. In The Structure of Attractors in Dynamical Systems. N.G. Markley, J.C. Martin, and W. Perrizo, editors. Springer-Verlag New York Inc., New York. 48-66.

22. Rosenstein, M.T., J.J. Collins, and C.J. De Luca. 1993. A practical method for calculating largest Lyapunov exponents from small data sets. Physica D. 65:117-134.

23. Bryant, P., R.V. Brown, and H.D. Abarbanel. 1990. Lyapunov exponents from observed time series. Phys. Rev. Lett. 65:1523-1526.

24. Kennel, M.B., R. Brown, and H.D.I. Abarbanel. 1992. Determining embedding dimension for phase-space reconstruction using a geometrical construction. Phys. Rev. A. 45:3403-3411.

25. Grassberger, P. 1986. Do climatic attractors exist? Nature (Lond.). 323: 609-612.

26. Theiler, J. 1986. Spurious dimension from correlation algorithms applied to limited time series data. Phys. Rev. A 34:2427-2429.

27. Eckmann, J., and D. Ruelle. 1992. Fundamental limitation for estimating dimensions and Lyapunov exponents in dynamical systems. Physica D 56: $185-187$.

28. Zhang, H., and A.V. Holden. 1995. Chaotic meander of spiral waves in the FitzHugh-Nagumo system. Chaos, Solitons \& Fractals. 5:661-760.

29. Gray, R.A., J. Jalife, A.V. Panfilov, W.T. Baxter, C. Cabo, J.M. Davidenko, and A.M. Pertsov. 1995. Mechanisms of cardiac fibrillation. Science (Wash. DC). 270:1222-1223.

30. Karma, A. 1994. Electrical alternans and spiral wave breakup in cardiac tissue. Chaos. 4:461-472.

31. Landau, L. 1944. On the problem of turbulence. Crit. Rev. Acad. Sci. 
URSS. 44:311-315

32. Ruelle, D., and F. Takens. 1971. On the nature of turbulence. Comm. Math. Phys. 20:167-192.

33. Newhouse, S., D. Ruelle, and F. Takens. 1978. Occurrence of strange Axiom-A attractors near quasiperiodic flow on $\mathrm{T}^{\mathrm{m}}, \mathrm{m} \geq 3$. Comm. Math. Phys. 64:35-40.

34. Brandstater, A. and H.L. Swinney. 1987. Strange attractors in weakly turbulent Couette-Taylor flow. Phys. Rev. A. 35:2207-2220.

35. Dubois, M., P. Berge, and V. Croquette. 1982. Study of non-steady convective regimes using Poincare sections. J. Physique-Lettres. 43:L-295-L-298.

36. Gollub, J.P., and H. Swinney. 1975. Onset of turbulence in a rotating fluid. Phys. Rev. Lett. 35:927-930.

37. Martin, S., and W. Martienssen. 1986. Circle maps and mode-locking in the driven electrical conductivity of barium sodium niobate crystals. Phys. Rev. Lett. 56:1522-1525.

38. Abraham, R.H., and C.D. Shaw. 1992. Dynamics: The Geometry of Behavior. Addison-Wesley, Redwood City, CA. 167-171.

39. Chakravarti, S., M. Marek, and W.H. Ray. 1995. Reaction-diffusion system with Brusselator kinetics - control of a quasiperiodic route of chaos. Phys. Rev. E. 52:2407-2423.

40. Sole, R.V., J. Valls, and J. Bascompte. 1992. Spiral waves, chaos, and multiple attractors in lattice models of interacting populations. Phys. Lett. A. 166:123-128.

41. Maselko, J., and K. Showalter. 1991. Chemical waves in inhomogeneous excitable media. Physica D. 49:21-32.

42. Lipp, P., and E. Niggli. 1993. Microscopic spiral waves reveal positive feedback in subcellular calcium signaling. Biophys. J. 65:2272-2276.

43. Lechleiter, J., S. Girard, E. Peralta, and D. Clapham. 1991. Spiral calcium wave propagation and annihilation in Xenopus laevis oocytes. Science (Wash. DC). 252:123-126.

44. Pertsov, A.M., J.M. Davidenko, R. Salomonsz, W.T. Baxter, and J. Jalife. 1993. Spiral waves of excitation underlie reentrant activity in isolated cardiac muscle. Circ. Res. 72:631-650.

45. Krinsky, V. 1966. Spread of excitation in an inhomogeneos medium. Biophysics. 11:776-784.

46. Tyson, J.J., and J.P. Keener. 1987. Spiral waves in a model of myocardium. Physica D. 29:215-222.

47. Courtemanche, M., and A.T. Winfree. 1991. Reentrant rotating waves in a Beeler-Reuter based model of two-dimensional cardiac electrical activity. Int. J. Bifurc. Chaos. 1:431-444.

48. Guevara, M.R., F. Alonso, D. Jeandupeux, and A.C.G. Ginneken. 1989. Alternans in periodically stimulated isolated ventricular myocytes: experiment and model. In Cell to Cell Signaling. A. Goldbeter, editor. Academic Press, Inc., New York. 551-563.

49. Nolasco, J.B., and R.W. Dahlen. 1968. A graphic method for the study of alternation in cardiac action potentials. J. Appl. Physiol. 25:191-196.

50. Barkley, D., M. Kness, and L.S. Tuckerman. 1990. Spiral wave dynamics in a simple model of excitable media. Phys. Rev. A. 42:2489-2492.

51. Karma, A. 1990. Meandering transition in two-dimensional excitable media. Phys. Rev. Lett. 65:2824-2827.

52. Courtemanche, M., L. Glass, and J. Keener. 1993. Instabilities of a propagating pulse in a ring of excitable media. Phys. Rev. Lett. 70:2182-2185.

53. Frame, L.H., and M.B. Simson. 1988. Oscillations of conduction, action potential duration, and refractoriness. A mechanism for spontaneous termination of reentrant tachycardias. Circulation. 78:1277-1287.

54. Kaplan, D.T., and R.J. Cohen. 1990. Is fibrillation chaos? Circ. Res. 67: $886-892$.

55. Witkowski, F.X., and P.A. Penkoske. 1990. Activation patterns during ventricular fibrillation. Ann. NY Acad. Sci. 591:219-231.

56. Karagueuzian, H.S., S.S. Khan, W. Peters, W.J. Mandel, and G.A. Diamond. 1990. Nonhomogeneous local atrial activity during acute atrial fibrillation: spectral and dynamic analysis. Pacing Clin. Electrophysiol. 13:1937-1942.

57. Nearing, B.D., A.H. Huang, and R.L. Verrier. 1991. Dynamic tracking of cardiac vulnerability by complex demodulation of the T wave. Science (Wash. DC). 252:437-440.

58. Rosenbaum, D.S., L.E. Jackson, J.M. Smith, H. Garan, J.N. Ruskin, and R.J. Cohen. 1994. Electrical alternans and vulnerability to ventricular arrhythmias. N. Engl. J. Med. 330:235-241.

59. Clusin, W.T., M.R. Bristow, D.S. Baim, J.S. Schroeder, P. Jaillon, P. Brett, and D.C. Harrison. 1982. The effects of diltiazem and reduced serum ionized calcium on ischemic ventricular fibrillation in the dog. Circ. Res. 50:518526

60. Lee, H.C., R. Mohabir, N. Smith, M.R. Franz, and W.T. Clusin. 1988. Effect of ischemia on calcium-dependent fluorescence transients in rabbit hearts containing indo 1 . Correlation with monophasic action potentials and contraction. Circulation. 78:1047-1059.

61. Nearing, B.D., J.J. Hutter, and R.L. Verrier. 1996. Potent antifibrillatory effect of combined blockade of calcium channels and 5- $\mathrm{HT}_{2}$ receptors with nexopamil during myocardial ischemia and reperfusion in dogs: comparison to diltiazem. J. Cardiovasc. Pharmacol. 27:777-787.

62. Konta, T., Y. Ikeda, M. Yamaki, K. Nakamura, K. Honma, I. Kubota, and S. Yasui. 1990. Significance of discordant ST alternans in ventricular fibrillation. Circulation. 82:2185-2189.

63. Christini, D.J., and J.J. Collins. 1996. Using chaos control and tracking to suppress a pathological nonchaotic rhythm in a cardiac model. Phys. Rev. E 53:R49-R52.

64. Garfinkel, A., M.L. Spano, W.L. Ditto, and J.N. Weiss. 1992. Controlling cardiac chaos. Science (Wash. DC). 257:1230-1235.

65. Kirchoff, C., F. Chorro, G.J. Scheffer, J. Brugada, K. Konings, Z. Zetelaki, and M. Allessie. 1993. Regional entrainment of atrial fibrillation studied by high-resolution mapping in open-chest dogs. Circulation. 88:736-749.

66. Cha, Y., U. Birgersdotter-Green, P.L. Wolf, B.B. Peters, and P. Chen 1994. The mechanism of termination of reentrant activity in ventricular fibrillation. Circulation. 74:495-506.

67. Shimizu, A., A. Nozaki, Y. Rudy, and A.L. Waldo. 1993. Characterization of double potentials in a functionally determined reentrant circuit. Multiplexing studies during interruption of atrial flutter in the canine pericarditis model. J. Am. Coll. Cardiol. 22:2022-2032. 